\title{
Effect of tip radius on the incipient plasticity of chromium studied by nanoindentation
}

\author{
D. $\mathrm{Wu}^{1}$, J. R. Morris ${ }^{2,1}$, and T. G. Nieh ${ }^{1, *}$ \\ ${ }^{1}$ Department of Materials Science and Engineering, University of Tennessee, Knoxville, Tennessee \\ 37996, USA \\ ${ }^{2}$ Materials Science and Technology Division, Oak Ridge National Laboratory, Oak Ridge, Tennessee \\ 37831, USA
}

* Please send all correspondence to Dr. T. G. Nieh

Email: tnieh@utk.edu

\begin{abstract}
The onset of plasticity in $\mathrm{Cr}$ was investigated by nanoindentation using indenters with tip radii ranging from 60 to $759 \mathrm{~nm}$. The stress for incipient plasticity was found to increase with decreasing tip radius. The cumulative pop-in probability on load could be described successfully by a combined model over the full range of tip radius, indicating that the incipient plasticity might be triggered either by the homogeneous nucleation of dislocation or by the activation of existing dislocations underneath the indenter.
\end{abstract}

Keywords: nanoindentation, onset of yielding, dislocation dynamics

In the last decade, an extrinsic size effect [1] has been intensively studied by deforming samples at the micro/nano scale. The yielding strengths of micro-pillars were found to depend upon the pillar diameter [2]. The strengthening was attributable to dislocation dynamics in small pillars such as dislocation multiplication and depletion at the pillar surface [3] and mechanical annealing [4]. Nanoindentation technique has also been widely employed to probe the size effect, for the size of plastic zone beneath the indenter can be regulated by 
the applied load and tip shape [5]. It is generally observed that the material strength increases with the decrease of sample size or test volume in the micro-nano dimension[1,6,7].

The incipient plasticity of pure metals (e.g. Al [5], Pt [8], Ni [9], Mg [10], W [11], Ta [12,13]) and alloys (e.g., a high entropy alloy FeCoCrNiMn [14] and $\mathrm{Ni}_{3} \mathrm{Al}$ [15] intermetallics) have been characterized by nanoindentation, and the tip radius used in these studies ranges from $113 \mathrm{~nm}$ to $1300 \mathrm{~nm}$, However, it is pointed out that, in each of these studies, no more than two tip radii were used, thus it is difficult to clarify the role of indenter tip radius.

Recently, Shim et al. [16] reported that the stress required for initiating the incipient plasticity in single-crystal $\mathrm{Ni}$ increased with the decrease of indenter radius. Whereas the smallest indenter produced reproducible results, larger indenter radii produced a strongly stochastic component in the pop-in strength. Assisted by MD simulation, Ngan et. al. [17] proposed a phenomenological model describing the stochastic pop-in behavior by nanoindentation in a given volume of material, but provided little quantitative analysis on the formation of dislocation. Bei et. al.[18] introduced a statistical model to interpret the cumulative probability of pop-in in Mo-3Nb alloy. However, the tail of statistical distribution for blunt indenter couldn't be understood in their theoretical framework. Morris et al. [19] showed that pop-ins could also be triggered by the activation of pre-existing dislocations under the indenter's stress field, and that a large spread of data occurred when the highly stressed region had a size comparable to typical dislocation spacing. When stress regions are large (due to large indenter radii), many dislocations contribute, and the spread of data is small. Conversely, when the radius is very small, the highly stressed region is unlikely to affect any dislocation, and a sharp distribution associated with homogeneous dislocation nucleation is expected. In an intermediate regime, where the length scale associated with the highly stressed region is close to the typical separation between defects, the distribution is 
broad. The statistics in that model accounted only for the stochastic nature of defects, and did not address the distribution associated with homogeneous nucleation. Shin et al. [20] revealed a power-law relationship between the critical shear stress and the tip radius in the blunt tip region, but the relationship, again, failed when a sharp tip was used. Most recently, the pop-in behavior of single-crystal Mo(001) [19] was reanalyzed [21] within the framework of the activation of pre-existing dislocations coupled with homogeneous dislocation nucleation. Though several studies on pure metals (Pt [8], Ni [9], and $\mathrm{Cr}$ [22]) and alloys $[14,15]$ also indicated that pop-in results from the nucleation process of dislocation at point defects, the dislocations are considered to nucleate homogeneously in the dislocation-free zone underneath a sharp indenter.

In the present study, we show that the pop-in stress in $\mathrm{Cr}$ depends on the tip radius of the indenter. A model combining two statistical models, one based on homogeneous dislocation nucleation in a dislocation-free crystal [8] (appropriate for the sharpest indenters) and another on pre-existing dislocations in the crystal [19], is proposed and shown to describe the pop-in cumulative probability in Cr over a broad range of tip radii.

The Cr sample was sliced from a coarse-grained $\mathrm{Cr}$ bulk prepared by arc melting method (purity > 99.99\%). The surface of the Cr sample was ground using 1200 and 2400grit $\mathrm{SiC}$, and then mechanically polished using alumina of $0.06 \mu \mathrm{m}$ in grit size. To reduce existing dislocations and minimize possible surface damage resulting from the mechanical polish, the Cr specimen was initially sealed in a fused silica tube filled with pure argon gas and, then, annealed at $1250{ }^{\circ} \mathrm{C}$ for $4 \mathrm{hr}$ [23]. As the final procedure, the annealed $\mathrm{Cr}$ was additionally electropolished in a solution $\left(\mathrm{H}_{3} \mathrm{PO}_{4}: \mathrm{H}_{2} \mathrm{SO}_{4}=4: 1\right)$ to remove a possible oxide layer resulting from annealing. The sample was immediately tested under nanoindentation. The grain size was determined to be about $0.5 \mathrm{~mm}$ by optical observation. 
Nanoindentation tests were performed using a Triboindenter (Hysitron Inc. Minneapolis, MN) equipped with diamond indenters with various tip radii. Single-crystal tungsten was used for calibration and the tip radii of the indenters used were determined to be $60.3 \pm 6.7 \mathrm{~nm}, 80.3 \pm 10.7 \mathrm{~nm}, 211 \pm 12 \mathrm{~nm}, 303 \pm 25 \mathrm{~nm}, 601 \pm 20 \mathrm{~nm}$ and $759 \pm 54 \mathrm{~nm}$. At least 50 indentation tests were performed with each indenter. For data consistence, tests were conducted on the same $\mathrm{Cr}$ grain at a loading rate of $2 \mathrm{mN} / \mathrm{s}$.

Typical load-displacement curves showing the pop-in event obtained from the indentation tests on $\mathrm{Cr}$ using the Berkovich indenters with the tip radii, $\mathrm{R}$, of 211 and $759 \mathrm{~nm}$. and cubic corner indenters $(\mathrm{R}=60 \mathrm{~nm}$ and $80 \mathrm{~nm})$ are shown in Figure 1. The elastic loading part of the P-h curve is well described by the Hertzian relation [24]: $P=4 E_{r} R^{0.5} h^{1.5} / 3$, where $R$ is the tip radius of the indenter, $h$ is the indentation depth, and $E_{r}$ is the reduced elastic modulus given by $E_{r}=\left[\left(\left(\begin{array}{ll}1 & 2\end{array}\right) / E\right)_{\text {Diamond }}+\left(\left(\begin{array}{ll}1 & 2\end{array}\right) / E\right)_{\mathrm{Cr}}\right]^{1}$, where the Poisson's ratio $v$ is 0.21 for $\mathrm{Cr}$ and 0.07 for diamond, and the elastic moduli are 279 and $1141 \mathrm{GPa}$ for $\mathrm{Cr}$ [25] and diamond [26], respectively. Due to a smaller tip radius of the cubic corner indenter, pop-in occurs at a much shallower depth $(<10 \mathrm{~nm})$.

In general, these pop-in events are triggered when the maximum shear stress $\tau_{\max }$ underneath the indenter exceeds a critical value. According to contact mechanics [24], $\tau_{\max }$ occurs at about half of the contact radius $a$ and is given by $\tau_{\max }=0.47\left[16 E_{r}^{2} P /\left(9 \pi^{3} R^{2}\right)\right]^{1 / 3}$, where $P$ is the applied load, and $R$ is the tip radius of the indenter. For an indenter, the contact radius is $a=\left(3 P R / 4 E_{r}\right)^{\frac{1}{3}}$.

According to the statistical analysis for a dislocation-free crystal [8], pop-in events is caused by the nucleation of dislocation assisted by stress, and the rate at which the pop-in events occur per material volume is given by $\dot{n}=\eta \exp [-(\varepsilon-\tau v) / k T]$, where $\varepsilon$ is the 
activation barrier for the pop-in event, $\tau$ is the applied stress over the activation volume $U$, and $\eta$ is the pre-exponential frequency factor. This must be integrated over the volume of stressed material underneath each indenter $(\Omega)$ to give the displacement burst rate. As a good approximation, the maximum shear stress $\tau_{\max }$ is assumed to be the stress bias $\tau$, and the sampling volume $\Omega$ is scaled with $a^{3}$, specifically $\Omega=3 K P R / 4 E_{r} \approx 3 \pi P R / 4 E_{r}$. Thus, the global pop-in rate $\dot{N}$ over the stressed volume is expressed as

$$
\dot{N}=\eta \cdot \iiint_{\Omega} \exp \left(-\frac{\varepsilon-\tau v}{k T}\right) d \Omega \approx \eta \cdot \frac{3 \pi P R}{4 E_{r}} \exp \left(-\frac{\varepsilon-\tau_{\max } v}{k T}\right)
$$

Following the Weibull statistics[27], the cumulative probability function $f$ obeys the equation: $d f / d t=(1-f) \dot{\mathrm{N}}(\mathrm{t})$ or in its integral form: $\ln [1-f(t)]=-\int_{0}^{t} \dot{N}\left(t^{\prime}\right) d t^{\prime}$. In the current study, the analytical form of $f(P)$ could be derived by combining Eq. (1) with the Weibull statistical equation (for details, see Ref. 24):

$$
f_{\text {nucleation }}(P)=1-\exp \left[-\frac{9 \pi R \eta}{4 E_{r} \dot{P} \alpha^{6}} \exp \left(-\frac{\varepsilon}{k T}\right) \beta\right]
$$

where $\alpha=0.47\left[16 E_{r}^{2} /\left(9 \pi^{3} R^{2}\right)\right]^{1 / 3} v / k T$ and $\beta$ is a complex but weak function [8] of load $P$.

The cumulative probability of pop-in $f$ is shown in Figure 2(a) as a function of applied load $P$ at various indenter tip radii. Each $f$ curve is noted to exhibit a "tail" toward the lowload side and the tail becomes more pronounced when a blunter tip is used. The tail is the region where the pop-in occurs at a lower pop-in load (or stress). Using the activation volume $v\left(0.287 \mathrm{~b}^{3}\right)$ and the activation energy $(0.505 \mathrm{eV})$ determined previously for the pop-in events in a coarse-grained $\mathrm{Cr}[22]$, the cumulative probability of pop-in $f$ can be calculated using the theoretical model [8] and directly compared with the experimental data. It is apparent in 
Figure 2(a) that the model predictions generally fit the data well, except a gradual deviation in the tail region. The deviation becomes more significant when the tip radius is larger.

Schuh's model [8] was based on the assumption that a material had no prior defects, in particular, dislocations. On the other hand, Morris et al. [19] proposed a statistical model to describe the stochastic behavior of nanoindentation pop-in in single crystals. However, his model was based on the fact that there were pre-existing defects, such as dislocations, FrankRead sources; thus, homogeneous dislocation nucleation was not necessary for the onset of plasticity. In this case, pop-in occurs when at least one defect is activated in the high-stressed volume of material beneath the indenter. The probability $q$ for the absence of the pop-in in the highly stressed region is predicted as $q=\exp \left[-\rho_{\text {defect }} V\left(\tau>\tau_{\text {pop-in }}\right)\right]$, where $\rho_{\text {defect }}$ is the density of pre-existing defect in the material, and $\tau_{\text {pop-in }}$ is the assumed stress required to activate a defect. $\tau_{\text {pop-in }}$ is assumed to be single-valued, for mathematical convenience, though more generally one would expect, which would depend on specific defect structure and orientation. Phani et al. [28] demonstrated that a similar model with orientation effects had only a minor effect on pop-in distribution. $V\left(\tau>\tau_{\text {pop-in }}\right)$ is the high-stressed volume where the local shear stress $\tau>\tau_{\text {pop-in }}$ in the stress field, in which the maximum shear stress $\tau_{\text {max }}$ beneath the indenter is determined by the tip radius $R$ and applied load $P$. As the applied load increases, a higher $\tau_{\max }$ is attained in the stressed zone and the stressed volume $V$ expands rapidly.

The functional relationship between $V$ and $\tau_{\max } / \tau_{\text {pop-in }}$ could be fitted in a power-law as

$$
V / a^{3}=-2.105 \times 10^{-4} x^{3}+0.128 x^{2}+5.695 x
$$

where $x=\left(\tau_{\max } / \tau_{\text {pop-in }}\right)-1$, as shown in Figure 3 . It is noted that the $V$ approaches zero as $x$ approaches zero. This power-law relation is valid within the range of material strength, namely, $\tau_{\max }<\mu / 2 \pi$. 
The cumulative probability for pop-in in Morris' model [19] is, therefore,

$$
f_{\text {activation }}=1-\exp \left[-\rho_{\text {defect }} V\left(\tau>\tau_{\text {pop-in }}\right)\right]
$$

For a material with fixed $\rho_{\text {defect }}$ and $\tau_{\text {pop-in, }}$ the $f$ function can be derived for any pairs of indenter tip radius $R$ and the maximum shear stress $\tau_{\max }$ below the indenter. Morris's model has been successfully applied to describe the pop-in behavior in single crystal Mo for blunt indenters $(R>3.75 \mu \mathrm{m})$, but showed large deviation for sharper indenters. When the tip radius decreases below $1.5 \mu \mathrm{m}$, the cumulative pop-in probability begins to converge to the limit of theoretical strength [19], suggesting a transition from dislocation activation to dislocation nucleation. It appears, therefore, a complete description of the cumulative pop-in probability over a broad range of tip radii should include both Schuh's and Morris' models. In the following, we present a model that combines these two models.

The survival probability for dislocation nucleation mechanism during indentation is $\exp \left\{-9 K R \eta \exp [-\varepsilon /(k T)] \beta /\left(4 E_{r} \dot{P} \alpha^{6}\right)\right\}($ Eq. (2)). When this combines with the survival probability $q$ for the activation of pre-existing defect, it leads to the total survival probability as $\exp \left\{-\rho_{\text {defect }} V-9 \pi R \eta \exp [-\varepsilon /(k T)] \beta /\left(4 E_{r} \dot{P} \alpha^{6}\right)\right\}$ during nanoindentation. Consequently, the total cumulative probability of pop-in is modified as

$$
f_{\text {total }}=1-\exp \left\{-\rho_{\text {defect }} V-\frac{9 \pi R \eta}{4 E_{r} \dot{P} \alpha^{6}} \exp \left(-\frac{\varepsilon}{k T}\right) \beta\right\}
$$

The modified model can apparently describe quite well the cumulative probability of pop-in at various tip radii, as shown in Figure 2(b). In this study, the pre-existing defects are considered pinned in the lattice, and a critical shear stress $\tau_{p o p-i n}$ is required for activation. For the modeling, the theoretical shear stress for the obstacle-controlled dislocation glide in 
single-crystal $\mathrm{Cr}(0.2 \mathrm{GPa}$ [29-31]) was used for the yield strength. A defect density of $\rho=1 \sim 1.5 \times 10^{17} \mathrm{~m}^{-3}$ is selected for the fitting, indicating that defect-driven pop-in will be important when the highly stressed region under the indenter has a volume of $\sim 1 / \rho\left(\sim 8 \mu \mathrm{m}^{3}\right)$. This density corresponds to a mean free distance of $2 \mu \mathrm{m}$ for defects. If we assume an equally spaced cubic array of dislocation lines, the dislocation density would be about $10^{11} \mathrm{~m}^{-2}$, consistent with the dislocation density value of $10^{10} \mathrm{~m}^{-2}$ determined in $\mathrm{Cr}$ [22] before.

Assume that the mean value of $\tau_{\max }$ is $\tau_{1 / 2}$, namely, the value of maximum shear stress when $f$ function is 0.5 , and insert $\alpha$, then for each radius, we have the following relationship between $\tau_{1 / 2}$ and the tip radius $R$ :

$$
\gamma \exp \left(-\frac{\varepsilon}{k T}\right) \beta R^{5}+\rho_{\text {defect }} V\left(\tau>\tau_{\text {pop-in }}\right)=\ln 2
$$

where $\gamma=264.2 \pi^{7} \eta(\mathrm{kT})^{6} /\left(E_{r}^{5} \dot{P} v^{6}\right)$.

From the experimental measurements, $\tau_{1 / 2}$ is plotted as a function of the indenter tip radius, as shown in Figure 4 . Evidently, $\tau_{1 / 2}$ decreases with increasing tip radius. To carry out the model prediction, the average activation parameters $\left(v=0.287 \mathrm{~b}^{3}, \varepsilon=0.505 \mathrm{eV}, \eta_{\text {ave }}=8.9\right.$ $\left.\times 10^{23} \mathrm{~s}^{-1} \mathrm{~m}^{-3}\right)$ and material parameters for $\mathrm{Cr}\left(b=0.25 \mathrm{~nm}, E_{r}=232.61 \mathrm{GPa}, \rho=1.5 \times 10^{17}\right.$ $\mathrm{m}^{-3}$ ) are inserted into Eq. (6). Evidently, the model prediction agrees well with the decreasing trend of critical shear stress with the tip radius as shown in Figure 4. The strength for the sharp indenter $(R=60 \mathrm{~nm})$ begins to deviate from the model prediction, because the upper bound of the theoretical strength is reached. On the other hand, when the tip radius is larger than $0.6 \sim 0.8 \mu \mathrm{m}$, the yielding strength begins to drop faster as a result of possible presence of pre-existing defect in the stressed volume. For the blunt tip $(R>1 \mu \mathrm{m})$, the predicted popin stress drops with tip radius following a power law of $\tau \sim R^{-2 / 3}$, which is consistent with the results reported in single-crystal $\operatorname{Mo}(100)[20]$. 
In summary, the stress required for incipient plasticity in $\mathrm{Cr}$ with a broad range of tip radii (from $60 \mathrm{~nm}$ to $759 \mathrm{~nm}$ ) was determined. The pop-in stress was found to increase with the reduction of tip radius and saturated at a tip radius less than $80 \mathrm{~nm}$. The cumulative probability of pop-in measured from using a sharp tip $(R<300 \mathrm{~nm})$ can be well described by model based on the assumption of testing a perfect crystal, originally proposed by Schuh et al [8]. However, data from using a blunt tip ( $\mathrm{R}>300 \mathrm{~nm})$ appear to fit better with a modified model assuming the test sample has pre-existing dislocations. This modified model, specifically, a combination of these two models, can predict the pop-in behavior in Cr over the entire range of tip radii, indicating a mechanistic transition from homogeneous dislocation nucleation to pre-existing defect activation when the tip radius increases. The transition is attributable to the fact that when the stressed volume underneath an indenter increases, the probability to encounter a dislocation also increases.

\section{Acknowledgement}

This work was performed under the NSF Contract DMR-0905979. JRM was supported by the Materials Sciences and Engineering Division, Office of Basic Energy Sciences (BES), U.S. Department of Energy (DOE).

\section{References}

[1] J.R. Greer, J.T.M. De Hosson, Prog. Mater. Sci. 56 (2011) 654.

[2] J.-Y. Kim, D. Jang, J.R. Greer, Acta Mater. 58 (2010) 2355.

[3] I. Ryu, W.D. Nix, W. Cai, Acta Mater. 61 (2013) 3233.

[4] L. Huang, Q.J. Li, Z.W. Shan, J. Li, J. Sun, E. Ma, Nat. Commun. 2 (2011).

[5] A. Barnoush, Acta Mater. 60 (2012) 1268.

[6] A.T. Jennings, M.J. Burek, J.R. Greer, Phys. Rev. Lett. 104 (2010) 135503.

[7] A.S. Schneider, D. Kaufmann, B.G. Clark, C.P. Frick, P.A. Gruber, R. Monig, O. Kraft, E. Arzt, Phys. Rev. Lett. 103 (2009). 
[8] J.K. Mason, A.C. Lund, C.A. Schuh, Phys. Rev. B 73 (2006) 54102.

[9] I. Salehinia, S.K. Lawrence, D.F. Bahr, Acta Mater. 61 (2013) 1421.

[10] H. Somekawa, C.A. Schuh, Acta Mater. 59 (2011) 7554.

[11] A.A. Zbib, D.F. Bahr, Metall. Mater. Trans. A 38 (2007) 2249.

[12] K. V Rajulapati, M.M. Biener, J. Biener, A.M. Hodge, Philos. Mag. Lett. 90 (2009) 35.

[13] M.M. Biener, J. Biener, A.M. Hodge, A. V Hamza, Phys. Rev. B 76 (2007) 165422.

[14] C. Zhu, Z. Lu, T. Nieh, Acta Mater. 61 (2013) 2993.

[15] P.C. Wo, L. Zuo, A.H.W. Ngan, J. Mater. Res. 20 (2005) 489.

[16] S. Shim, H. Bei, E.P. George, G.M. Pharr, Scr. Mater. 59 (2008) 1095.

[17] A.H.W. Ngan, L. Zuo, P.C. Wo, Proc. R. Soc. A Math. Phys. Eng. Sci. 462 (2006) 1661 .

[18] H. Bei, Y.F. Gao, S. Shim, E.P. George, G.M. Pharr, Phys. Rev. B 77 (2008) 60103.

[19] J.R. Morris, H. Bei, G.M. Pharr, E.P. George, Phys. Rev. Lett. 106 (2011) 165502.

[20] C. Shin, S. Shim, J. Mater. Res. 27 (2012) 2161.

[21] T.L. Li, H. Bei, J.R. Morris, E.P. George, Y.F. Gao, Mater. Sci. Technol. 28 (2012) 1055 .

[22] D. Wu, T.G. Nieh, Mater. Sci. Eng. A 609 (2014) 110.

[23] A.C. McLaren, Aust. J. Phys. 17 (1964) 447.

[24] K.L. Johnson, Contact Mechanics, Cambridge University Press, Cambridge, 1985.

[25] D.R. Askeland, The Science and Engineering of Materials, 3rd ed., PWS, Boston (MA), 1994.

[26] G. Simmons, H. Wang, Single Crystal Elastic Constants and Calculated Aggregate Properties, 2nd editio, The MIT Press, Cambridge, 1971.

[27] W. Weibull, J. Appl. Mech. 18 (1951) 293.

[28] P. Sudharshan Phani, K.E. Johanns, E.P. George, G.M. Pharr, J. Mater. Res. 28 (2013) 2728.

[29] D. Wu, J. Zhang, J.C. Huang, H. Bei, T.G. Nieh, Scr. Mater. 68 (2013) 118.

[30] C.N. Reid, A. Gilbert, G.T. Hahn, Trans. Metall. Soc. Aime 239 (1967) 467. 
[31] Harold J Frost and Michael F Ashby, Deformation-Mechanism Maps, The Plasticity and Creep of Metals and Ceramics, Pergamon Press, Cambridge University UK, 1982.

Captions for figures in the manuscripts

Figure. 1 Typical load-displacement curves from nanoindentation tests on annealed $\mathrm{Cr}$ using indenters with various tip radii. The elastic part of loading curves is well predicted by the Hertzian relation.

Figure 2 The cumulative probability of pop-in events underneath indentation with various tip radii. Solid lines are predictions based on (a) a homogeneous dislocation nucleation model [8] and (b) a model combining homogeneous dislocation nucleation and the activation of preexisting dislocation processes. For larger radii, significant deviations from the homogeneous dislocation nucleation model occur, indicating the importance of heterogeneous nucleation and activation.

Figure. 3 The stressed volume $V$ underneath an indenter and its variation with $\tau_{\max } / \tau_{\text {pop-in }}$ according to the Hertzian theory.

Figure. 4 Comparison of experimental data with the prediction of modified model in the $\tau_{1 / 2^{-}}$ $\mathrm{R}$ relationship (Eq. (6)) 


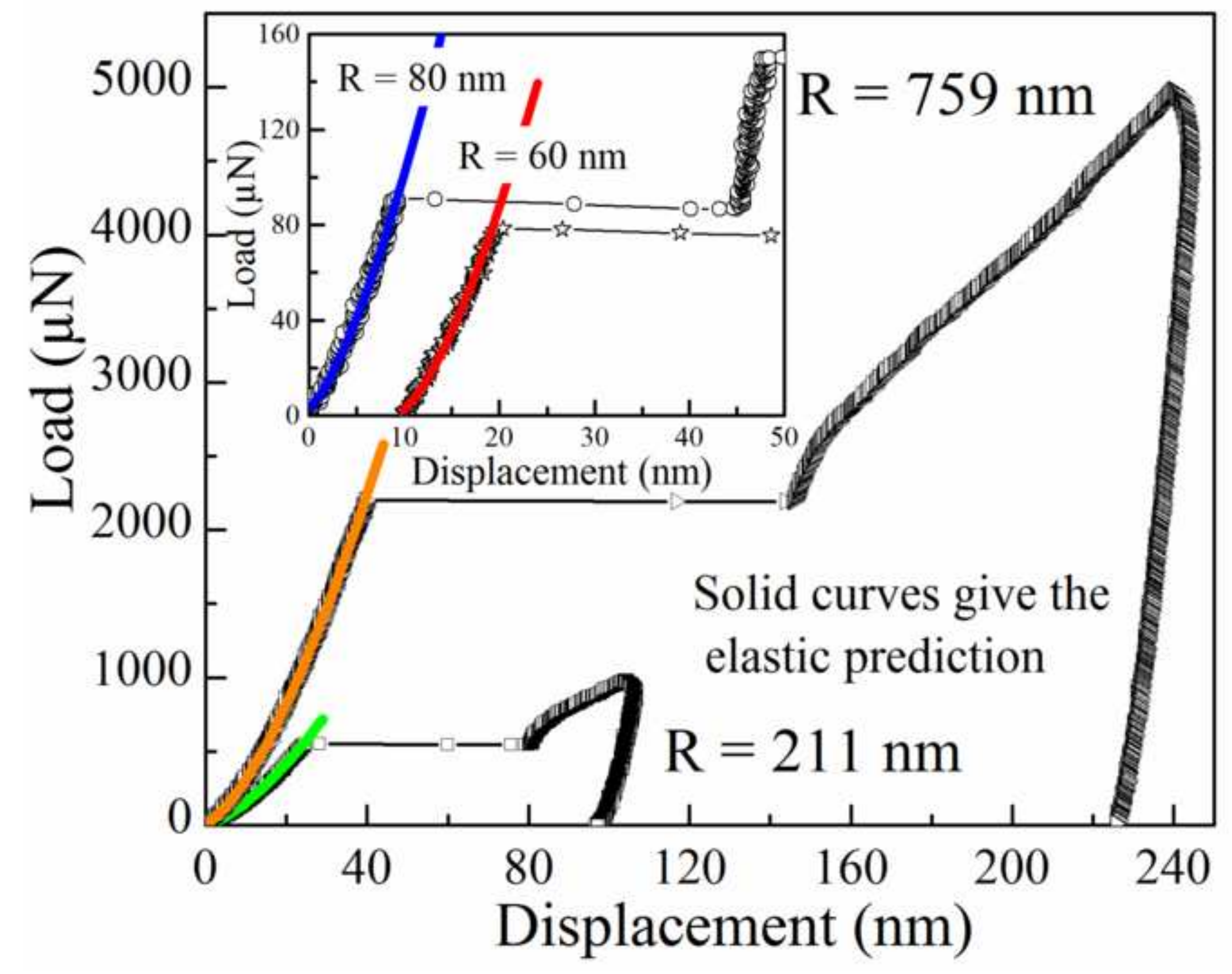




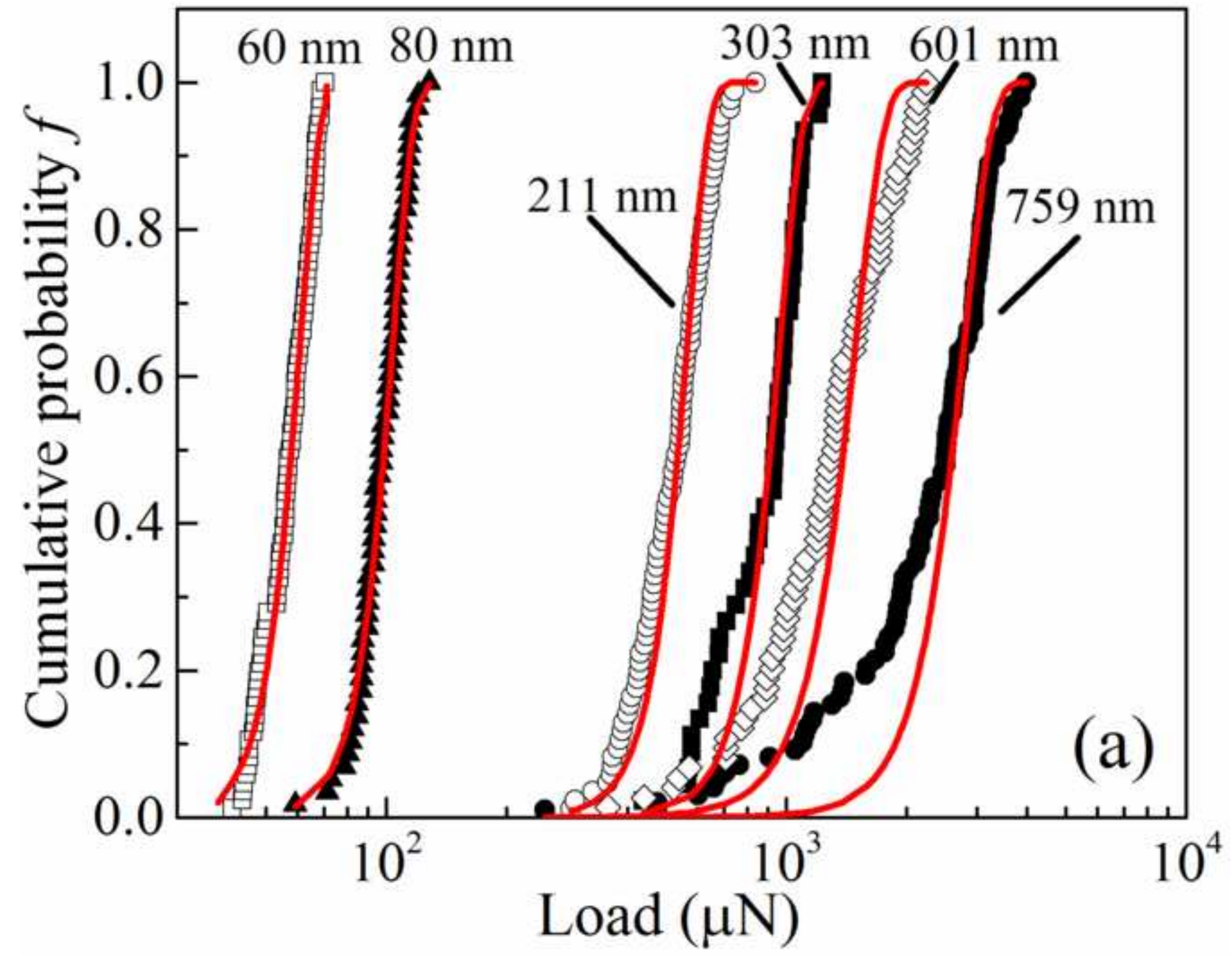




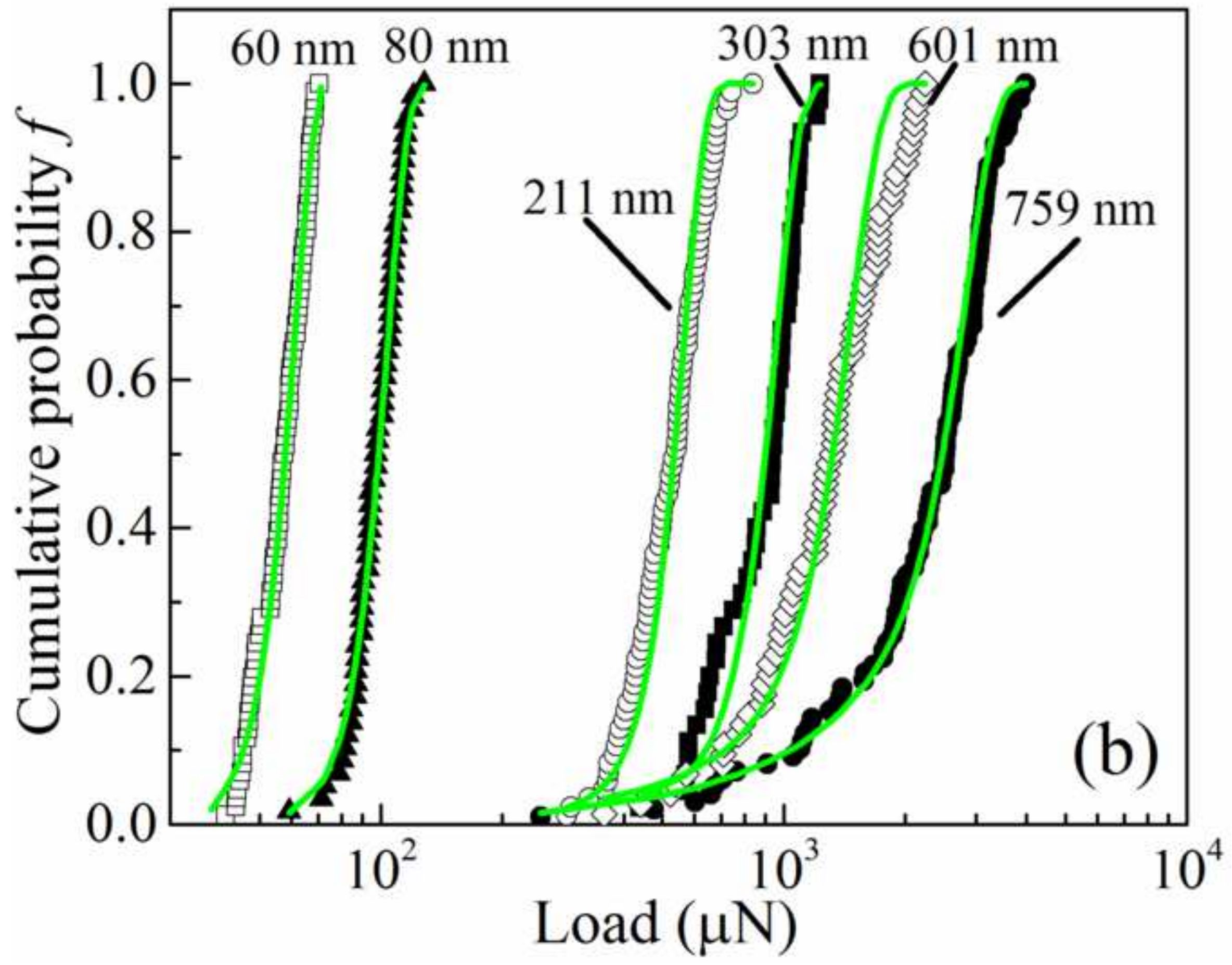




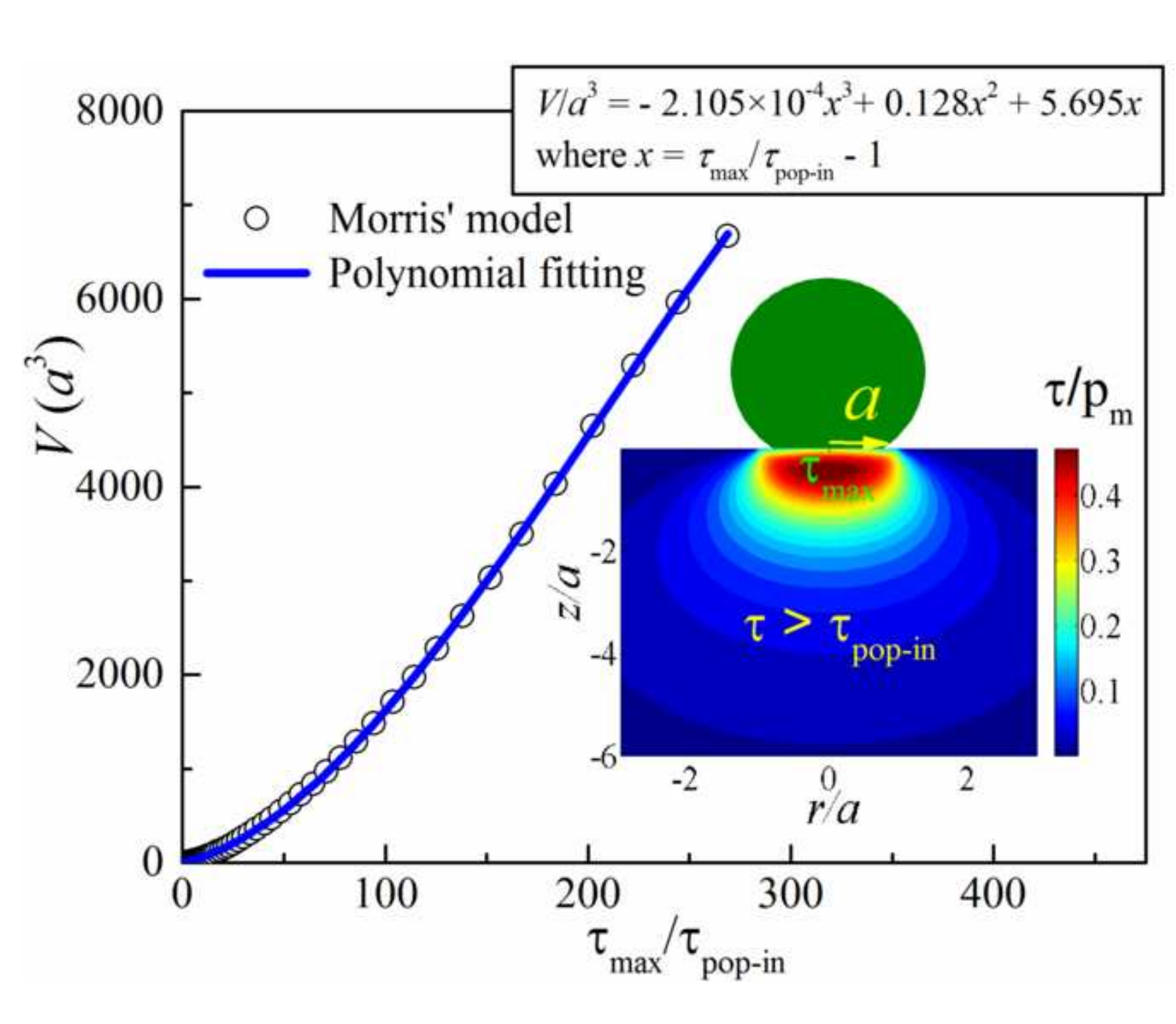

$$
\text { . }
$$

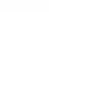

.

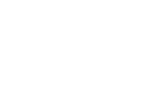
. 


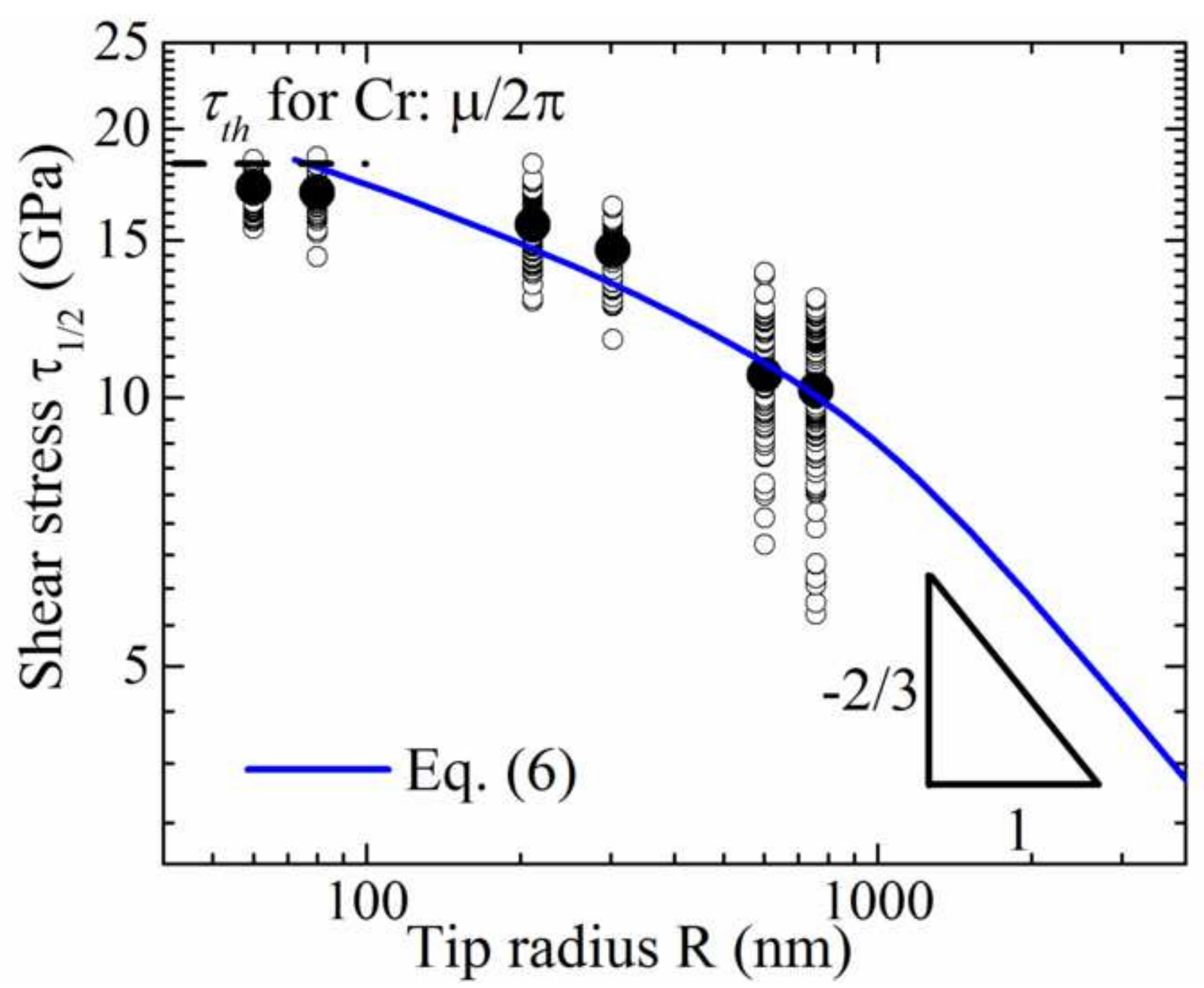

